\title{
Prediction for the Cosmological Constant and Constraints on SUSY GUTS: Status Report for Resummed Quantum Gravity
}

\author{
B.F.L. Ward ${ }^{* \dagger}$ \\ Baylor University, Waco, TX, USA \\ E-mail: BEL_Wardabaylor.edu
}

\begin{abstract}
We give a status report on the theory of resummed quantum gravity. We recapitulate the use of our resummed quantum gravity approach to Einstein's general theory of relativity to estimate the value of the cosmological constant as $\rho_{\Lambda}=(0.0024 \mathrm{eV})^{4}$. The estimate is made in the context of the Planck scale cosmology formulation of Bonanno and Reuter. We discuss the constraints on susy GUT models that follow from the closeness of the estimate to experiment. Various consistency checks on the calculation are addressed and we use the Heisenberg uncertainty principle to remove a large part of the remaining uncertainty in our estimate of $\rho_{\Lambda}$.
\end{abstract}

ICHEP2018, 39th International Conference on High Energy Physics

5-11 July 2018

Seoul, S. Korea

\footnotetext{
* Speaker.

${ }^{\dagger}$ On Research Leave from Baylor University, Waco, TX, USA, Jan. 4 - Jul. 31,2018, at Werner-Heisenberg-Institut, Max-Planck-Institut fuer Physik, Muenchen, Germany
} 


\section{Introduction}

We start with the basic question which our title engenders, "What is resummation in quantum field theory?" For comparison we illustrate "summation" with the elementary example:

$$
\frac{1}{1-x}=\sum_{n=0}^{\infty} x^{n}
$$

The geometric series is summed to infinity to yield the analytic result that is well-defined except for a pole at $x=1$. Indeed, even though the mathematical tests for convergence of the series would only guarantee convergence for $|x|<1$, the result of the summation yields a function that is well-defined in the entire complex plane except for the simple pole at $x=1$. We see that infinite order summation can yield behavior very much improved from what one sees order-by-order in the series.

We are thus motivated to resum series that are already being summed to seek improvement in our knowledge of the represented function. We illustrate this as follows:

$$
\sum_{n=0}^{\infty} C_{n} \alpha_{s}^{n}\left\{\begin{array}{l}
=F_{\mathrm{RES}}\left(\alpha_{s}\right) \sum_{n=0}^{\infty} B_{n} \alpha_{s}^{n}, \text { EXACT } \\
\cong G_{\mathrm{RES}}\left(\alpha_{s}\right) \sum_{n=0}^{\infty} \tilde{B}_{n} \alpha_{s}^{n}, \text { APPROX. }
\end{array}\right.
$$

The original Feynman series for a process under study is on the LHS. Two versions of resumming this original series are on the RHS. One, labeled exact, is an exact re-arrangement of the original series. The other, labeled approx., only agrees with the LHS to some fixed order N in the expansion parameter $\alpha_{s}$. Which version is to be preferred has been discussed for some time [1]. A related more general discussion now occurs for quantum gravity.

We may ask whether quantum gravity is even calculable in relativistic quantum field theory? There are various answers. String theory [2] argues the answer is no, the true fundamental theory entails a one-dimensional Planck scale superstring. Loop quantum gravity [3] also argues the answer is no, the fundamental theory entails a space-time foam with a Planck scale loop structure. The Horava-Lifshitz theory [4] also argues the answer is no because the fundamental theory requires Planck scale anisoptropic scaling for space and time. Kreimer [5] suggests that quantum gravity is leg-renormalizable, so that the answer is yes. Weinberg [6] suggests that quantum gravity may be asymptotically safe, with an S-matrix that depends only on a finite number of observable parameters, due to the presence of a non-trivial UV fixed point, with a finite dimensional critical surface; this amounts to an answer of yes. The authors in Refs. [7, 8, 9, 10, 11, 12], using Wilsonian [13] field-space exact renormalization group methods, obtain results which support Weinberg's UV fixed-point. Ref. [14] also gives support to Weinberg's asymptotic safety suggestion.

In what follows, we extend the YFS $[15,16]$ version $^{1}$ of the exact example to resum the Feynman series for the Einstein-Hilbert Lagrangian for quantum gravity. Just as we see in the example in eq.(1.1), the resultant resummed theory, resummed quantum gravity (RQG), is very much better behaved in the UV compared to what one would estimate from that Feynman series. We have shown $[18,19,20,21]$ that the RQG realization of quantum gravity leads to Weinberg's UV-fixed-point behavior for the dimensionless gravitational and cosmological constants and that the resummed theory is actually UV finite. RQG and the latter results are reviewed in Section 2.

We show further that the RQG theory, taken together with the Planck scale inflationary $[24,25]$ cosmology formulation in Refs. $[22,23]^{2}$ from the asymptotic safety approach to quantum gravity in Refs. [7, 8, 9, 10, 11, 12], allows us to predict [27] the cosmological constant $\Lambda$. Due to the prediction's closeness to the observed value [28, 29], we discuss its reliability and argue [30] that its uncertainty is at the level of a factor of $O(10)$. There follow constraints on susy GUT's. The Planck scale cosmology that we use and the latter results are presented in Section 3.

\section{Overview of Resummed Quantum Gravity}

The Standard Theory ${ }^{3}$ of elementary particles contains many point particles. To explore their graviton interactions, we treat spin as an inessential complication [32] and consider the Higgs-

\footnotetext{
${ }^{1}$ YFS-type soft resummation and its extension to quantum gravity was also worked-out by Weinberg in Ref. [17].

${ }^{2}$ The authors in Ref. [26] also proposed the attendant choice of the scale $k \sim 1 / t$ used in Refs. [22, 23].

${ }^{3}$ We follow D.J. Gross [31] and call the Standard Model the Standard Theory henceforth.
} 
gravition extension of the Einstein-Hilbert theory, already studied in Refs. [33, 34], as exemplary:

$$
\begin{aligned}
& \mathcal{L}(x)=-\frac{1}{2 \kappa^{2}} R \sqrt{-g}+\frac{1}{2}\left(g^{\mu \nu} \partial_{\mu} \varphi \partial_{\nu} \varphi-m_{o}^{2} \varphi^{2}\right) \sqrt{-g} \\
& =\frac{1}{2}\left\{h^{\mu v, \lambda} \bar{h}_{\mu v, \lambda}-2 \eta^{\mu \mu^{\prime}} \eta^{\lambda \lambda^{\prime}} \bar{h}_{\mu_{\lambda}, \lambda} \eta^{\sigma \sigma^{\prime}} \bar{h}_{\mu^{\prime} \sigma, \sigma^{\prime}}\right\} \\
& +\frac{1}{2}\left\{\varphi_{, \mu} \varphi^{, \mu}-m_{o}^{2} \varphi^{2}\right\}-\kappa h^{\mu v}\left[\overline{\varphi_{, \mu} \varphi_{, \nu}}+\frac{1}{2} m_{o}^{2} \varphi^{2} \eta_{\mu v}\right] \\
& -\kappa^{2}\left[\frac{1}{2} h_{\lambda \rho} \bar{h}^{\rho \lambda}\left(\varphi_{, \mu} \varphi^{, \mu}-m_{o}^{2} \varphi^{2}\right)-2 \eta_{\rho \rho^{\prime}} h^{\mu \rho} \bar{h}^{\rho^{\prime} v} \varphi_{, \mu} \varphi_{, v}\right]+\cdots \text {. }
\end{aligned}
$$

$R$ is the curvature scalar, $g$ is the determinant of the metric of space-time $g_{\mu v} \equiv \eta_{\mu v}+2 \kappa h_{\mu v}(x)$, and $\kappa=\sqrt{8 \pi G_{N}}$. We expand [33,34] about Minkowski space with $\eta_{\mu v}=\operatorname{diag}\{1,-1,-1,-1\} . \varphi(x)$ is the physical Higgs field as our representative scalar field for matter and $\varphi(x)_{, \mu} \equiv \partial_{\mu} \varphi(x)$. We have introduced Feynman's notation $\bar{y}_{\mu v} \equiv \frac{1}{2}\left(y_{\mu \nu}+y_{v \mu}-\eta_{\mu v} y_{\rho}{ }^{\rho}\right)$ for any tensor $y_{\mu v}{ }^{4}$. The bare (renormalized) scalar boson mass here is $m_{o}(m)$ and we set presently the small observed [28, 29] value of the cosmological constant to zero so that our quantum graviton, $h_{\mu v}$, has zero rest mass. Feynman $[33,34]$ has essentially worked out the Feynman rules for $(2.1)$, including the rule for the famous Feynman-Faddeev-Popov [33, 35, 36] ghost contribution required for unitarity with the fixing of the gauge (we use the gauge in Ref. [33], $\partial^{\mu} \bar{h}_{\nu \mu}=0$ ).

As we have shown in Refs. [18, 19, 20], the large virtual IR effects in the respective loop integrals for the scalar propagator in quantum general relativity can be resummed to the exact result $i \Delta_{F}^{\prime}(k)=\frac{i}{k^{2}-m^{2}-\Sigma_{s}(k)+i \varepsilon}=\frac{i e_{g}^{\prime \prime}(k)}{k^{2}-m^{2}-\Sigma_{s}^{\prime}+i \varepsilon}$ for $\left(\Delta=k^{2}-m^{2}\right)$ where $B_{g}^{\prime \prime}(k)=\frac{\kappa^{2}\left|k^{2}\right|}{8 \pi^{2}} \ln \left(\frac{m^{2}}{m^{2}+\left|k^{2}\right|}\right)$. The form for $B_{g}^{\prime \prime}(k)$ holds for the UV(deep Euclidean) regime ${ }^{5}$, so that $\left.\Delta_{F}^{\prime}(k)\right|_{\text {resummed }}$ falls faster than any power of $\left|k^{2}\right|$. See Ref. [18] for the analogous result for $\mathrm{m}=0$. Here, $-i \Sigma_{s}(k)$ is the 1PI scalar self-energy function so that $i \Delta_{F}^{\prime}(k)$ is the exact scalar propagator. The residual $\Sigma_{s}^{\prime}$ starts in $O\left(\kappa^{2}\right)$. We may drop it in calculating one-loop effects. When the respective analogs of $\left.i \Delta_{F}^{\prime}(k)\right|_{\text {resummed }}{ }^{6}$ are used for the elementary particles, all quantum gravity loops are UV finite $[18,19,20]$.

Specifically, we extend our resummed propagator results to all the particles in the ST Lagrangian and to the graviton itself and show in the Refs. $[18,19,20]$ that the denominator for the propagation of transverse-traceless modes of the graviton becomes $\left(M_{P l}\right.$ is the Planck mass)

$$
q^{2}+\Sigma^{T}\left(q^{2}\right)+i \varepsilon \cong q^{2}-q^{4} \frac{c_{2, e f f}}{360 \pi M_{P l}^{2}},
$$

where $c_{2, \text { eff }} \cong 2.56 \times 10^{4}$ is defined in Refs. $[18,19,20]$. We thus get (we use $G_{N}$ for $G_{N}(0)$ )

$$
G_{N}(k)=G_{N} /\left(1+\frac{c_{2, e f f} k^{2}}{360 \pi M_{P l}^{2}}\right), g_{*}=\lim _{k^{2} \rightarrow \infty} k^{2} G_{N}\left(k^{2}\right)=\frac{360 \pi}{c_{2, e f f}} \cong 0.0442 .
$$

For the dimensionless cosmological constant $\lambda_{*}$ we isolate [27] $\Lambda$ via the VEV of Einstein's equation $G_{\mu \nu}+\Lambda g_{\mu \nu}=-\kappa^{2} T_{\mu \nu}$ in a standard notation. We find that a scalar makes the contribution to $\Lambda$ given by ${ }^{7} \Lambda_{s} \cong-8 \pi G_{N}\left[\frac{1}{G_{N}^{2} 64 \rho^{2}}\right]$ and that a Dirac fermion contributes -4 times $\Lambda_{s}$ to $\Lambda$, where

\footnotetext{
${ }^{4}$ Our conventions for raising and lowering indices in the second line of (2.1) are the same as those in Ref. [34].

${ }^{5}$ By Wick rotation, the identification $-\left|k^{2}\right| \equiv k^{2}$ in the deep Euclidean regime gives immediate analytic continuation to the result for $B_{g}^{\prime \prime}(k)$ when the usual $-i \varepsilon, \varepsilon \downarrow 0$, is appended to $m^{2}$.

${ }^{6}$ These follow from the spin independence $[17,18,37]$ of a particle's coupling to the graviton.

${ }^{7}$ We note the use here in the integrand of $2 k_{0}^{2}$ rather than the $2\left(\vec{k}^{2}+m^{2}\right)$ in Ref. [21], to be consistent with $\omega=$ -1 [38] for the vacuum stress-energy tensor.
} 
$\rho=\ln \frac{2}{\lambda_{c}}$ with $\lambda_{c}(j)=\frac{2 m_{j}^{2}}{\pi M_{P l}^{2}}$ for particle j with mass $m_{j}$. The deep UV limit of $\Lambda$ then becomes, allowing $G_{N}(k)$ to run,

$$
\Lambda(k) \underset{k^{2} \rightarrow \infty}{\longrightarrow} k^{2} \lambda_{*}, \lambda_{*}=-\frac{c_{2, e f f}}{2880} \sum_{j}(-1)^{F_{j}} n_{j} / \rho_{j}^{2} \cong 0.0817
$$

where $F_{j}$ is the fermion number of particle $j, n_{j}$ is the effective number of degrees of freedom of $j$ and $\rho_{j}=\rho\left(\lambda_{c}\left(m_{j}\right)\right) . \lambda_{*}$ vanishes in an exactly supersymmetric theory .

The UV fixed-point calculated here, $\left(g_{*}, \lambda_{*}\right) \cong(0.0442,0.0817)$, and the estimate $\left(g_{*}, \lambda_{*}\right) \approx$ $(0.27,0.36)$ in Refs. [22, 23] are similar in that in both of them $g_{*}$ and $\lambda_{*}$ are positive and are less than 1 in size. See Refs. [18] for further discussion of the relationship between the two fixed-point predictions.

\section{Review of Planck Scale Cosmology and an Estimate of $\Lambda$}

In the Einstein-Hilbert theory, the authors in Ref. [22, 23], using the exact renormalization group for the Wilsonian [13] coarse grained effective average action in field space, as discussed in Section 1, have argued that the dimensionless Newton and cosmological constants approach UV fixed points as the attendant scale $k$ goes to infinity in the deep Euclidean regime, as we have also found in RQG. To make contact with cosmology, one may use a connection between the momentum scale $k$ characterizing the coarseness of the Wilsonian graininess of the average effective action and the cosmological time $t$. From this latter connection, the authors in Refs. [22, 23] arrive at the following extension of the standard cosmological equations:

$$
\left(\frac{\dot{a}}{a}\right)^{2}+\frac{K}{a^{2}}=\frac{1}{3} \Lambda+\frac{8 \pi}{3} G_{N} \rho, \dot{\rho}+3(1+\omega) \frac{\dot{a}}{a} \rho=0, \dot{\Lambda}+8 \pi \rho \dot{G}_{N}=0, G_{N}(t)=G_{N}(k(t)), \Lambda(t)=\Lambda(k(t)) .
$$

Here, $\rho$ is the density and $a(t)$ is the scale factor with the Robertson-Walker metric given as

$$
d s^{2}=d t^{2}-a(t)^{2}\left(\frac{d r^{2}}{1-K r^{2}}+r^{2}\left(d \theta^{2}+\sin ^{2} \theta d \phi^{2}\right)\right)
$$

where $K=0,1,-1$ correspond respectively to flat, spherical and pseudo-spherical 3-spaces for constant time t. The equation of state is $p(t)=\omega \rho(t)$, where $p$ is the pressure. The relationship between $k$ and the cosmological time $t$ is $k(t)=\frac{\xi}{t}$ for a constant $\xi>0$ determined from constraints on physical observables.

The authors in Refs. [22, 23], using the UV fixed points for $k^{2} G_{N}(k) \equiv g_{*}$ and $\Lambda(k) / k^{2} \equiv \lambda_{*}$ obtained independently, solve the cosmological system given above. For $K=0$, they find a solution in the Planck regime where $0 \leq t \leq t_{\text {class }}$, with $t_{\text {class }}$ a "few" times the Planck time $t_{P l}$, which joins smoothly onto a solution in the classical regime, $t>t_{\text {class }}$, which coincides with standard Friedmann-Robertson-Walker phenomenology but with the horizon, flatness, scale free HarrisonZeldovich spectrum, and entropy problems all solved purely by Planck scale quantum physics. We now review how to use the Planck scale cosmology of Refs. [22,23] and the UV limits $\left\{g_{*}, \lambda_{*}\right\}$ in RQG [18, 19, 20] in Refs. [21] to predict [27] the current value of $\Lambda$.

Specifically, the Planck scale cosmology description of inflation in Ref. [23] gives the transition time between the Planck regime and the classical Friedmann-Robertson-Walker(FRW) regime as $t_{t r} \sim 25 t_{P l}$. Starting with the quantity $\rho_{\Lambda}\left(t_{t r}\right) \equiv \frac{\Lambda\left(t_{t r}\right)}{8 \pi G_{N}\left(t_{t r}\right)}$ we show in Ref. [27] that we get, employing the arguments in Refs. [39] ( $t_{e q}$ is the time of matter-radiation equality),

$$
\begin{aligned}
\rho_{\Lambda}\left(t_{0}\right) & \cong \frac{-M_{P l}^{4}\left(1+c_{2, e f f} k_{t r}^{2} /\left(360 \pi M_{P l}^{2}\right)\right)^{2}}{64} \sum_{j} \frac{(-1)^{F} n_{j}}{\rho_{j}^{2}} \times \frac{t_{t r}^{2}}{t_{e q}^{2}} \times\left(\frac{t_{e q}^{2 / 3}}{t_{0}^{2 / 3}}\right)^{3} \\
& \cong \frac{-M_{P l}^{2}(1.0362)^{2}\left(-9.194 \times 10^{-3}\right)}{64} \frac{(25)^{2}}{t_{0}^{2}} \cong\left(2.4 \times 10^{-3} e V\right)^{4} .
\end{aligned}
$$


$t_{0} \cong 13.7 \times 10^{9} \mathrm{yrs}$ is the age of the universe. The estimate in (3.3) is close to the experimental result $\left.[29]^{8} \rho_{\Lambda}\left(t_{0}\right)\right|_{\text {expt }} \cong\left((2.37 \pm 0.05) \times 10^{-3} \mathrm{eV}\right)^{4}$.

The three issues of the effect of various spontaneous symmetry breaking energies on $\Lambda$, the effect of our approach to $\Lambda$ on big bang nucleosynthesis(BBN) [41], and the effect of the time dependence of $\Lambda$ and $G_{N}$ on the covariance $[42,43,44]$ of the theory are discussed in detail in Ref. [27]. We refer the reader to the discussions in Ref. [27], respectively. Concerning the issue of the error on our estimate, we have argued in Ref. [30] that the Heisenberg uncertainty principle, taken together with the structure of the solutions of Einstein's equation, implies the constraint $k \geq \frac{\sqrt{5}}{2 w_{0}}=\frac{\sqrt{5}}{2} \frac{1}{\sqrt{3 / \Lambda(k)}}$ where $\Lambda(k)$ follows from (3.3) (see Eq.(52) in Ref. [27]). This constraint's equality gives the estimate [27] of the transition time, $t_{\mathrm{t} r}=\alpha / M_{P l}=1 / k_{\mathrm{tr}}$, from the Planck scale inflationary regime $[22,23]$ to the Friedmann-Robertson-Walker regime via the implied value of $\alpha$. When we solve for $\alpha$ we get $\alpha \cong 25.3$, which agrees with the value $\alpha \cong 25$ implied by the numerical studies in Ref. [22, 23]. This agreement implies an error on $t_{\text {tr }}$ at the level of a factor $O(3)$ or less and an uncertainty on $\Lambda$ reduced from a factor of $O(100)$ [27] to a factor of $O(10)$.

One may ask what would happen to our estimate if there were a susy GUT theory at high scales? For definiteness and purposes of illustration, in Ref. [27] we use the susy SO(10) GUT model in Ref. [45] to illustrate how such a theory might affect our estimate of $\Lambda$. We show that one needs a very high mass for the gravitino or that one needs twice the usual particle content with the susy partners of the new quarks and leptons at masses much lower than their partners' masses - see Ref. [27]. We thank Profs. S. Bethke and W. Hollik for the support and kind hospitality of the Werner-Heisenberg-Institut, MPI, Munich, Germany.

\section{References}

[1] F. Berends, private communication, ICHEP 1988 Conference Dinner, Munich, Germany, 1988.

[2] M.B. Green and J. H. Schwarz, Phys. Lett. B149 (1984) 117; ibid. 151 (1985) 21 (1985); D.J. Gross et al., Phys. Rev. Lett. 54 (1985) 502; Nucl. Phys. B256 (1985) 253; ibid. 267 (1986) 75; see also M. Green, J. Schwarz and E. Witten, Superstring Theory, v. 1 and v. 2, (Cambridge U. Press, Cambridge, 1987); J. Polchinski, String Theory, v. 1 and v. 2, (Cambridge U. Press, Cambridge, 1998).

[3] See for example V.N. Melnikov, Gravit. Cosmol. 9 (2003) 118; L. Smolin, hep-th/0303185; A. Ashtekar and J. Lewandowski, Class. Quantum Grav. 21 (2004) R53; A. Perez, gr-qc/0409061; M. Bojowald et al., arXiv.org:0806.3929, and references therein.

[4] P. Horava, Phys. Rev. D 79 (2009) 084008.

[5] D. Kreimer, Ann. Phys. 323 (2008) 49; ibid. 321 (2006) 2757.

[6] S. Weinberg, in General Relativity, an Einstein Centenary Survey, eds. S. W. Hawking and W. Israel, (Cambridge Univ. Press, Cambridge, 1979).

[7] M. Reuter, Phys. Rev. D57 (1998) 971, and references therein.

[8] O. Lauscher and M. Reuter, Phys. Rev. D66 (2002) 025026.

[9] E. Manrique, M. Reuter and F. Saueressig, Ann. Phys. 326 (2011) 44, and references therein.

[10] A. Bonanno and M. Reuter, Phys. Rev. D62 (2000) 043008.

[11] D. F. Litim, Phys. Rev. Lett.92(2004) 201301; Phys. Rev. D64 (2001) 105007; P. Fischer and D.F. Litim, Phys. Lett. B638 (2006) 497 and references therein.

[12] D. Don and R. Percacci, Class. Quant. Grav. 15 (1998) 3449; R. Percacci and D. Perini, Phys. Rev. D67(2003) 081503; ibid.68 (2003) 044018; R. Percacci, ibid.73(2006) 041501; A. Codello, R. Percacci and C. Rahmede, Int.J. Mod. Phys. A23(2008) 143.

[13] K. G. Wilson, Phys. Rev. B4 (1971) 3174, 3184; K. G. Wilson, J.Kogut, Phys. Rep. 12 (1974) 75; F. Wegner, A. Houghton, Phys. Rev. A8(1973) 401; S. Weinberg, "Critical Phenomena for Field Theorists”, Erice Subnucl. Phys. (1976) 1; J. Polchinski, Nucl. Phys. B231 (1984) 269.

\footnotetext{
${ }^{8}$ The analysis in Ref. [40] also gives a value for $\rho_{\Lambda}\left(t_{0}\right)$ that is qualitatively similar to this experimental result.
} 
[14] J. Ambjorn et al., Phys. Lett. B690 (2010) 420.

[15] D. R. Yennie, S. C. Frautschi, and H. Suura, Ann. Phys. 13 (1961) 379; see also K. T. Mahanthappa, Phys. Rev. 126 (1962) 329, for a related analysis.

[16] S. Jadach and B.F.L. Ward, Phys. Rev. D38 (1988) 2897;ibid. D39 (1989) 1471; ibid. D40 (1989) 3582; Comp. Phys. Commun. 56 (1990) 351; S.Jadach, B.F.L. Ward and Z. Was, Comput. Phys. Commun. 66 (1991) 276; ibid. 79 (1994) 503; ibid. 124 (2000) 233; ibid.130 (2000) 260; Phys. Rev.D63 (2001) 113009; S.Jadach, W. Placzek and B.F.L. Ward, Phys. Lett. B390 (1997) 298; S. Jadach et al., Phys. Lett. B417 (1998) 326; Comput. Phys. Commun. 119 (1999) 272; ibid.140 (2001) 432, 475; Phys. Rev. D61 (2000) 113010; ibid. D65 (2002) 093010.

[17] S. Weinberg, Phys. Rev. 140 (1965) B 516.

[18] B.F.L. Ward, Open Nucl.Part.Phys.Jour. 2(2009) 1.

[19] B.F.L. Ward, Mod. Phys. Lett. A17 (2002) 237.

[20] B.F.L. Ward, Mod. Phys. Lett. A19 (2004) 143.

[21] B.F.L. Ward, Mod. Phys. Lett. A23 (2008) 3299.

[22] A. Bonanno and M. Reuter, Phys. Rev. D65 (2002) 043508.

[23] A. Bonanno and M. Reuter, Jour. Phys. Conf. Ser. 140 (2008) 012008, and references therein.

[24] A. H. Guth and D.I. Kaiser, Science 307 (2005) 884; A. H. Guth, Phys. Rev. D23 (1981) 347.

[25] A. Linde, Lect. Notes. Phys. 738 (2008) 1.

[26] I. L. Shapiro and J. Sola, Phys. Lett. B475 (2000) 236.

[27] B.F.L. Ward, Phys. Dark Univ. 2 (2013) 97.

[28] A.G. Riess et al., Astron. Jour. 116 (1998) 1009; S. Perlmutter et al., Astrophys. J. 517 (1999) 565.

[29] C. Amsler et al., Phys. Lett. B667 (2008) 1.

[30] B.F.L. Ward, Mod. Phys. Lett. A 30 (2015) 1550206.

[31] D.J. Gross, talk in SM@50 Symposium, Case Western Reserve University, Cleveland, OH, June, 2018.

[32] M.L. Goldberger, private communication.

[33] R.P. Feynman, Acta Phys. Pol.24 (1963) 697-722.

[34] R.P. Feynman, Lectures on Gravitation, eds. F. Moringo and W. Wagner, (Caltech, Pasadena, 1971).

[35] L.D. Faddeev and V.N. Popov, "Perturbation theory for gauge invariant fields", preprint ITF-67-036, NAL-THY-57 (translated from Russian by D. Gordon and B.W. Lee).

[36] Faddeev LD and Popov VN. Feynman diagrams for the Yang-Mills field. Phys Lett B 1967; 25: 29-30.

[37] S. Weinberg, The Quantum Theory of Fields, v.1,(Cambridge University Press, Cambridge, 1995).

[38] Ya. B. Zeldovich, Sov. Phys. Uspekhi 11 (1968) 381.

[39] V. Branchina and D. Zappala, G. R. Gravit. 42 (2010) 141; arXiv:1005.3657, and references therein.

[40] J. Sola, J. Phys. A41 (2008) 164066.

[41] See for example G. Stiegman, Ann. Rev. Nucl. Part. Sci.57 (2007) 463, and references therein.

[42] S. Basilakos, M. Plionis and J. Sola, arXiv:0907.4555.

[43] J. Grande et al., J. Cos. Astropart. Phys. 1108 (2011) 007.

[44] H. Fritzsch and J. Sola, arXiv:1202.5097, and references therein.

[45] P.S. Bhupal Dev and R.N. Mohapatra, Phys. Rev. D82 (2010) 035014, and references therein. 